PSICOLOGÍA

IBEROAMERICANA
Psicología Iberoamericana ISSN: 1405-0943

revista.psicologia@ibero.mx

Universidad Iberoamericana, Ciudad de México México

\title{
Prácticas parentales y consumo de marihuana en universitarios
}

Betancourt Ocampo, Diana; González González, Alejandro; Cota Flores, Diane Anahí Fátima; García Ponce, Diana; Hernández Rendón, Karla; Salazar Mera, Marcela

Prácticas parentales y consumo de marihuana en universitarios

Psicología Iberoamericana, vol. 26, núm. 1, 2018

Universidad Iberoamericana, Ciudad de México, México

Disponible en: http://www.redalyc.org/articulo.oa?id=133959553005 


\title{
Prácticas parentales y consumo de marihuana en universitarios
}

Parental practices and consumption of marijuana in university students

\author{
Diana Betancourt Ocampo diana.betancourt@anahuac.mx \\ Univerisdad Anáhauc, México \\ Alejandro González González \\ Univerisdad Anáhauc, México \\ Diane Anahí Fátima Cota Flores \\ Univerisdad Anáhauc, México \\ Diana García Ponce \\ Univerisdad Anáhauc, México \\ Karla Hernández Rendón \\ Univerisdad Anáhauc, México \\ Marcela Salazar Mera \\ Univerisdad Anáhauc, México
}

Resumen: El presente estudio buscó determinar las diferencias en las prácticas parentales por consumo de marihuana en jóvenes universitarios. Se seleccionó una muestra no probabilística de 583 estudiantes universitarios ( $53 \%$ fueron mujeres y $47 \%$ hombres), con un promedio de edad de 21.33 años. Se adaptó la Escala de prácticas parentales de Andrade y Betancourt (2008), la cual se conforma de dos subescalas (una para la madre y otra para el padre), que evalúan: imposición, comunicación, autonomía, control psicológico y control conductual. Además, se utilizaron seis indicadores para evaluar el consumo de marihuana. Los resultados mostraron que $47.16 \%$ de los estudiantes ha consumido marihuana alguna vez en su vida. Asimismo, se encontraron diferencias significativas en las prácticas parentales por categoría de consumo, donde los jóvenes que no han consumido percibieron en general prácticas parentales más positivas respecto a los que han consumido en el último año y en el último mes.

Palabras clave: marihuana, universitarios, prácticas parentales, estudiantes, padres.

Abstract: The present study determines the differences in parental practices for marijuana use among university students. A non-probabilistic sample of 583 university students ( $53 \%$ were women and $47 \%$ men) was selected, with a mean age of 21.33 years. The Parental Practices scale of Andrade and Betancourt (2008) was adapted, which consists of two subscales (one for the mother and one for the father), which evaluate: imposition, communication, autonomy, psychological control and behavioral control. In addition, six indicators were used to assess marijuana use. The results showed that $47.16 \%$ of students have used marijuana sometime in their lifetime. Likewise, significant differences were found in parental practices by consumption category, where young people who did not consume marijuana experienced more positive parental practices compared to those who had consumed in the last year and in the last month.

Keywords: marijuana, university students, parental practices, students, parents.

\section{INTRODUCCIÓN}

De acuerdo con el Informe Mundial sobre Drogas 2016, la proporción de personas que han consumido drogas ilegales a nivel mundial se 
ha mantenido constante desde el año 2011 hasta el 2014 (5.2\% de la población las ha consumido), con la marihuana, además, como la sustancia de mayor preferencia; donde en el 2014 aproximadamente 183 millones de personas reportaron el consumo de dicha sustancia (unodc, 2016).

En nuestro país, datos de la Encuesta Nacional de Consumo de Drogas, Alcohol y Tabaco 2016-2017 (encodat) muestran un incremento en el consumo de marihuana de alguna vez en la vida de las mediciones del 2002 al 2016 en población de 12 a 65 años de edad. Según la encuesta, para el año 2002 el consumo de marihuana era de $3.5 \%$ en la población general, mientras que para el año 2016 incrementó a 8.6\%. Al hacer el análisis por sexo, los hallazgos indican el mismo patrón de incremento en ambos, ya que en el caso de los varones el incremento fue de 6.9\% a $14 \%$, mientras que para las mujeres fue de $0.7 \%$ a $3.7 \%$. Este mismo patrón se sigue para el indicador de consumo en el último año, donde en el caso de los hombres fue de $1.2 \%$, en la medición del 2002, a 3.5\% para el 2016; mientras que para las mujeres, el incremento fue de $0.1 \%$ a $0.9 \%$. Cuando se realiza el análisis en el grupo de 18 a 34 años de edad en la medición del 2016, los resultados indican que $12.8 \%$ de esta población indicó que alguna vez en la vida ha consumido marihuana; al reportar los resultados por sexo, éstos indican que 20\% de los hombres y $6.1 \%$ de las mujeres de este grupo poblacional han consumido marihuana alguna vez en su vida. Sobre el indicador de consumo en el último año, los hallazgos mostraron que $3.5 \%$ de la población de 18 a 34 años la han consumido (5.9\% en los hombres y $1.3 \%$ en mujeres) (Villatoro-Velázquez at al., 2017). Específicamente en población joven en nuestro país existen pocos estudios que muestran la incidencia del consumo de drogas ilegales en ese grupo de edad, particularmente en población universitaria; sin embargo, de acuerdo con un estudio realizado por Villatoro et al. (2009) el 34.7\% de estudiantes de licenciatura de una muestra representativa de una institución pública reportaron que han consumido marihuana alguna vez en su vida.

Por otro lado, diferentes estudios han mostrado que el consumo de marihuana se asocia con diversos factores: individuales y contextuales; respecto a los primeros, se encuentran los rasgos de personalidad (Costa, 2015; Szerman \& Peris, 2008), una alta búsqueda de sensaciones y de impulsividad (Bravo de Medina, Echeburúa, \& Aizpiri, 2010; Polaino \& De las Heras, 2001), una baja asertividad y una inadecuada toma de decisiones (Moreno, 2003; Palacios, Fuertes, \& Cabrear, 2001), el consumo de tabaco y alcohol a edades más tempranas (Iglesias, Cavada, Silva, \& Cáceres, 2007; Moreno, 2003), problemas con el consumo de alcohol, una baja autoestima y sintomatología depresiva (CogolloMilanés et al., 2011).

En cuanto a los contextuales, la literatura sugiere que la accesibilidad que los jóvenes tienen para adquirir la droga puede ser un factor que facilite su consumo (Castellón, 2006; Polaino \& De las Heras, 2001; nida, 2015). Asimismo, se ha documentado que los pares juegan un papel relevante en el consumo de sustancias no sólo ilegales sino también 
legales, (Hernández, 2007; nida, 2015). Algunos otros estudios han documentado el rol que juega la familia en el consumo de sustancias, entre lo que se ha reportado está que un ambiente familiar disfuncional puede ser considerado un factor de riesgo para el consumo de marihuana (Cogollo-Milanés et al., 2011). Como se puede apreciar, son diversos los factores que se vinculan al consumo de marihuana; sin embargo, el contexto familiar sigue siendo uno de los factores más relevantes. Uno de los campos estudiados en el área familiar es el que se refiere a las prácticas parentales, las cuales de acuerdo con Smetana (2000) se refirieren a los mecanismos que utilizan directamente los padres hacia las metas de socialización del hijo, las cuales pueden clasificarse en tres grandes categorías: apoyo, control conductual y control psicológico.

El control conductual se refiere a las conductas que realizan los padres buscando regular, supervisar y controlar la conducta del hijo(a); un componente importante en este tipo de control es el conocimiento de los padres sobre la ubicación, las actividades y los compañeros del joven o adolescente (Brown, Mounts, Lamborn, \& Steinberg, 1993; Kerr \& Stattin, 2000; Patterson, Reid, \& Dishion, 1992). Específicamente sobre la asociación de esta práctica parental y el consumo de marihuana, Lac y Crano (2009) realizaron un meta-análisis con el que encontraron una relación fuerte entre este tipo de control y el bajo consumo de marihuana en población adolescente, es por ello que los autores sugieren que el monitoreo parental puede considerarse como un factor protector para el consumo de marihuana; además, los autores encontraron efectos significativos por sexo, donde en las muestras de mujeres fue mayor el tamaño del efecto que en los varones.

En este mismo sentido, Lac et al. (2011) llevaron a cabo un estudio donde examinaron la relación entre factores familiares (monitoreo parental, comunicación padre-hijo y cohesión familiar) y el consumo de marihuana en una muestra de adolescentes latinos. Los resultados mostraron que los tres factores familiares evaluados fueron predictores del consumo, no obstante se encontró una relación más fuerte con el monitoreo parental; además, se reportan efectos significativos por sexo, donde las mujeres parecen tener un relación más fuerte entre las variables estudiadas que los hombres.

King, Vidourek y Merianos (2015) llevaron a cabo un estudio con población adolescente donde analizaron cómo algunas conductas de control conductual parental (por ejemplo, revisar que hayan realizado sus tareas escolares y dentro de casa, el monitorear la cantidad de tiempo que veían la televisión, etcétera) podrían incrementar el riesgo de consumo de marihuana en el último año y en el último mes. Los resultados mostraron efectos significativos de las diferentes conductas de control parental sobre su consumo (último año y último mes).

Por otro lado, el apoyo parental se define como el grado en que los hijos se sienten aceptados y tomados en cuenta por sus padres y que ha mostrado una relación positiva con el bienestar de los hijos (Maccoby $\&$ Martin, 1983). Cordova et al. (2014) realizaron un estudio donde analizaron las trayectorias del funcionamiento familiar y del consumo 
de sustancias en estudiantes de bachillerato, los autores encontraron una asociación entre el bajo apoyo parental y el incremento de riesgo de consumo de sustancias, entre ellas la marihuana. Por otro lado, Luk, Patock-Peckham y King (2015) realizaron una investigación donde analizan el papel del apoyo, la autonomía y la imposición parental sobre el consumo de alcohol, tabaco y marihuana en población universitaria, los resultados específicamente sobre marihuana mostraron que sólo el apoyo materno se asoció de manera negativa con el consumo de marihuana.

Johnson, McBride, Hopkins y Pepper (2014) examinaron el vínculo padre-hijo asociado al consumo de sustancias en adolescentes. Los autores incluyeron tanto la comunicación, la percepción del apoyo parental y el monitoreo como parte de la evaluación del vínculo padre-hijo. Los resultados del estudio mostraron correlaciones negativas entre un vínculo padre-hijo y el consumo de sustancias, es decir, aquellos adolescentes que reportaron puntajes altos en comunicación, apoyo y monitoreo parental son quienes se involucran con menor frecuencia en el consumo de sustancias, entre las que se encuentra la marihuana. En este mismo sentido, Becoña et al. (2013) reportaron que los adolescentes que perciben un menor control (conductual) por parte de la madre y altos puntajes de afecto tanto del padre como de la madre tienen más posibilidades de consumir marihuana, estos efectos los encontraron más consistentes en las mujeres que en los varones.

Por lo que respecta al control psicológico, Barber (1996) lo define como un tipo de control intrusivo dentro del intento de los padres por manipular la conducta, la identidad y el desarrollo psicológico del joven o adolescente. En general, la literatura previa ha mostrado que este tipo de control se asocia con mayor frecuencia con problemáticas más de corte emocional que con problemáticas conductuales, como lo es el consumo de sustancias; sin embargo, Kincaid, Jones, Cuellar y Gonzalez (2011) realizaron un estudio donde evaluaron la relación entre el control conductual y el control psicológico sobre problemas internalizados, externalizados y el involucramiento en conductas de riesgo en población adolescente, cabe señalar que dentro del consumo de sustancias sólo evalúan el consumo de alcohol. Los autores encontraron que el control psicológico fue una variable predictora sólo para los problemas internalizados y externalizados pero no para las conductas de riesgo; en el caso del control conductual sí se encontró una relación significativa con el involucramiento en conductas de riesgo.

Con base en lo anterior, el propósito del presente estudio fue determinar las diferencias en las prácticas parentales por consumo de marihuana en jóvenes universitarios. La hipótesis que se busca probar es que los jóvenes que perciben un alto control conductual y apoyo parental y bajos puntajes en control psicológico son aquellos que no han consumido marihuana o lo han hecho de manera experimental. 


\section{MÉTODO}

Se realizó un estudio no experimental, transversal, descriptivocorrelacional.

\section{Participantes}

Se seleccionó una muestra no probabilística de 583 estudiantes universitarios, de los cuales $54.4 \%$ pertenecían a instituciones privadas y $45.6 \%$ a instituciones públicas; de ellos $53 \%$ fueron mujeres y $47 \%$ hombres, de entre 17 y 45 años de edad, $(M=21.33$, $\mathrm{DE}=2.27)$. Los participantes eran estudiantes de diferentes licenciaturas: Ingeniería y Actuaria (25.4\%), Psicología y Pedagogía (23.2\%), Derecho (14.4\%), Comunicación y Diseño (11.1\%), Medicina, Nutrición y Odontología (10.8\%), Turismo y Gastronomía (7\%), Humanidades y Artes (4.1\%), Negocios y Economía (3.9\%). Cabe señalar que el único criterio de inclusión de los participantes fue que fueran estudiantes de las instituciones a las que se acudió.

\section{Instrumento}

Se adaptó la Escala de Prácticas Parentales de Andrade y Betancourt (2008), debido a que dicho instrumento fue diseñado originalmente para población adolescente. El instrumento original consta de dos subescalas: una que evalúa las conductas de la madre (40 reactivos) y otra para las del padre ( 40 reactivos), cada una de las subescalas se conforma con cinco dimensiones: comunicación, imposición, control psicológico, control conductual y autonomía; es una escala tipo Likert con cuatro opciones de respuesta (de nunca a siempre). La adaptación del instrumento consistió en agregar seis reactivos en la dimensión de comunicación en cada una de las subescalas (madre/padre) (por ejemplo, "Le puedo platicar de mi relación con mi novio(a)", "Me pregunta a qué se dedican mis amigos"). Para analizar las propiedades psicométricas del instrumento adaptado se realizó un estudio piloto previo donde los resultados mostraron índices de confiabilidad y validez adecuados. Las dos subescalas quedaron conformadas por cinco dimensiones cada una: imposición $(\alpha=.937$ para el papá y $\alpha=.794$ para la mamá), comunicación $(\alpha=.921$ para el papá y $\alpha=.891$ para la mamá), autonomía ( $\alpha=.897$ para el papá y $\alpha=.903$ para la mamá), control psicológico $(\alpha=.821$ para el papá y $\alpha=.808$ para la mamá) y control conductual ( $\alpha=.772$ para el papá y $\alpha=.915$ para la mamá).

Además, se utilizaron seis indicadores para evaluar el consumo de marihuana, los cuales evaluaban: (1) consumo alguna vez en la vida, (2) edad de inicio del consumo, (3) consumo en el último mes, (4) consumo en el último año, (5) forma de consumo y (6) número de ocasiones que han consumido (Villatoro et al., 2009). 


\section{Procedimiento}

La aplicación del instrumento se realizó en seis universidades (públicas y privadas) del área metropolitana de la Ciudad de México y de la ciudad de Toluca, el instrumento fue autoaplicable, de manera individual y en algunos casos de forma grupal. A todos los participantes se les explicó el objetivo de la investigación y se les solicitó su participación voluntaria, obteniendo su consentimiento de manera verbal. El tiempo aproximado que se tardaron los participantes en responder el instrumento fue de 15 a 20 minutos. En el caso de las aplicaciones grupales se cuidó que los participantes no observaran las respuestas de los otros jóvenes para cuidar la privacidad de las respuestas.

\section{RESULTADOS}

\section{Consumo de maribuana}

Para determinar la distribución de los participantes en los diferentes indicadores que evaluaron el consumo de marihuana se realizaron análisis de frecuencias (para el total de la muestra) y pruebas Ji Cuadrada para analizar las diferencias por sexo y por tipo de universidad (pública y privada). Como se observa en la Tabla 1 , los resultados indicaron que cerca de la mitad de los jóvenes de los cuales se obtuvo información reportaron que alguna vez en su vida han consumido marihuana; al hacer los análisis por sexo, se observó que es significativamente $(\mathrm{X} 2=36.92$, $\mathrm{p}<.001)$ mayor el porcentaje de los varones que informaron que han consumido esta sustancia en comparación con las mujeres. Cuando se les preguntó por el consumo dentro de los 12 meses previos al estudio, aproximadamente una cuarta parte del total de los jóvenes expresaron que han consumido marihuana; del mismo modo que en el indicador anterior, hay una ligera proporción mayor de hombres que informan que han consumido marihuana en el último año en contraste con las mujeres; sin embargo no se encontraron diferencias significativas ( $\mathrm{X} 2=2.50, \mathrm{p}>.05)$. Sobre el consumo en el último mes, cerca de $15 \%$ del total de los jóvenes reportaron que han consumido marihuana, con una proporción significativamente mayor $(\mathrm{X} 2=9.29, \mathrm{p}>.05)$ de hombres que de mujeres.

En cuanto al número de ocasiones que han consumido marihuana en toda su vida, tanto para el total de la muestra como para el grupo de las estudiantes, el mayor porcentaje informó que la ha consumido de una a dos ocasiones; los resultados mostraron diferencias en la distribución del número de ocasiones por el sexo de los estudiantes $(\mathrm{X} 2=9.52, \mathrm{p}>.05)$, donde llamó la atención que en cuanto a los varones, se encontraron las mismas proporciones tanto para la categoría de 3 a 5 y de 6 a 10 ocasiones. Respecto a la forma de consumo, la mayor parte de los universitarios (por total y por sexo) indicaron que han consumido la marihuana en cigarro; en segundo lugar se ubicó el consumo de esta sustancia con pipa. Los hallazgos en cuanto a las diferencias por sexo y por forma de 
consumo mostraron que no había diferencias significativas $(p>.05)$ en casi todas las categorías, excepto en el consumo por vaporizador, donde fue significativamente $(\mathrm{X} 2=8.62, \mathrm{p}>.01)$ mayor la proporción de hombres que mencionó que ha consumido marihuana por esta forma en contraste con las mujeres (ver Tabla 1 ).

Por lo que se refiere al promedio de edad del primer consumo, los hallazgos indicaron que éste fue aproximadamente a los 17 años (ver Tabla 1), se realizó una prueba $t$ de Student para determinar si había diferencias significativas entre hombre y mujeres, los resultados mostraron que no había diferencias por sexo [ $\mathrm{t}(274)=1.31$, $\mathrm{p}>.05]$.

Al hacer el análisis de los indicadores de consumo de marihuana por tipo de institución educativa (pública y privada) los resultados mostraron que fue significativamente mayor $(\mathrm{X} 2=11.43, \mathrm{p}>.01)$ el porcentaje de estudiantes de universidades privadas que informaron que han consumido marihuana alguna vez en su vida, en contraste con las públicas, lo cual, se encontró como tendencia para el consumo en el último año y en el último mes, sin embargo no se encontraron diferencias significativas ( $p>.05)$ (ver Tabla 2). Respecto al número de ocasiones que han consumido dicha sustancia, los hallazgos mostraron que para el caso de los jóvenes que estudian en instituciones públicas casi una tercera parte mencionó que lo ha realizado de 3 a 5 ocasiones; para los jóvenes de universidades privadas una cuarta parte indicó que ha consumido marihuana de entre 6 y 10 veces, en este indicador no se encontraron diferencias significativas ( $p>.05$ ) por tipo de institución. 
Tabla 1

\begin{tabular}{|c|c|c|c|}
\hline \multicolumn{4}{|c|}{$\begin{array}{l}\text { Indicadores de consumo de marihuana por el total de los } \\
\text { participantes y por sexo }\end{array}$} \\
\hline & $\begin{array}{c}\text { Total } \\
\%\end{array}$ & $\begin{array}{c}\text { Hombres } \\
\%\end{array}$ & $\begin{array}{c}\text { Mujeres } \\
\%\end{array}$ \\
\hline $\begin{array}{c}\text { Han consumido } \\
\text { marihuana alguna vez } \\
\text { en la vida }\end{array}$ & 47.16 & 56.93 & 38.51 \\
\hline $\begin{array}{c}\text { Han consumido } \\
\text { marihuana en el último } \\
\text { año }\end{array}$ & 25.55 & 33.21 & 18.77 \\
\hline $\begin{array}{c}\text { Han consumido } \\
\text { marihuana en el último } \\
\text { mes }\end{array}$ & 14.92 & 19.34 & 11 \\
\hline \multicolumn{4}{|l|}{$\begin{array}{c}\text { Número de ocasiones } \\
\text { de consumo de } \\
\text { marihuana }\end{array}$} \\
\hline \multicolumn{4}{|l|}{ De 1 a 2 ocasiones } \\
\hline De 3 a 5 ocasiones & 25.9 & 19.2 & 34.7 \\
\hline De 6 a 10 ocasiones & 23.7 & 24.4 & 22.9 \\
\hline De 11 a 49 ocasiones & 23 & 24.4 & 21.2 \\
\hline \multirow[t]{2}{*}{ De 50 a más ocasiones } & 17.2 & 19.9 & 13.6 \\
\hline & 10.2 & 12.2 & 7.6 \\
\hline \multicolumn{4}{|l|}{$\begin{array}{l}\text { Forma de consumo de } \\
\text { marihuana }\end{array}$} \\
\hline Cigarro & 81.5 & 85.3 & 76.5 \\
\hline Pipa & 47.6 & 44.9 & 51.3 \\
\hline Hashish & 23.6 & 25.6 & 21 \\
\hline Bong & 26.2 & 30.1 & 21 \\
\hline $\begin{array}{l}\text { Ingerido (brownies, } \\
\text { pasteles, etcétera) }\end{array}$ & 42.5 & 39.7 & 43.2 \\
\hline Vaporizador & 21.8 & 28.2 & 13.4 \\
\hline Otra & 4 & 3.9 & 4.2 \\
\hline $\begin{array}{l}\text { Edad del primer } \\
\text { consumo }\end{array}$ & $\begin{array}{l}M=17.36 \\
(D E=2.56)\end{array}$ & $\begin{array}{c}M=17.19 \\
(D E=2.62)\end{array}$ & $\begin{array}{l}M=17.60 \\
(D E=2.48)\end{array}$ \\
\hline
\end{tabular}

Tabla 1

Indicadores de consumo de marihuana por el total de los participantes y por sexo

Por lo que se refiere a la forma de consumo, llamó la atención que en contraste con las comparaciones por sexo, al hacer la comparación por tipo de institución educativa se encontraron diferencias significativas en la mayoría de las formas de consumo (excepto en las categorías de consumo ingerido y en otras opciones), en cuanto al consumo de marihuana en cigarro, éste fue el método con mayor porcentaje de consumo, estos resultados aplican tanto para los estudiantes de universidades públicas como privadas, sin embargo fue mayor la proporción en las instituciones privadas $(\mathrm{X} 2=10.85, \mathrm{p}>.001)$. 
Tabla 2

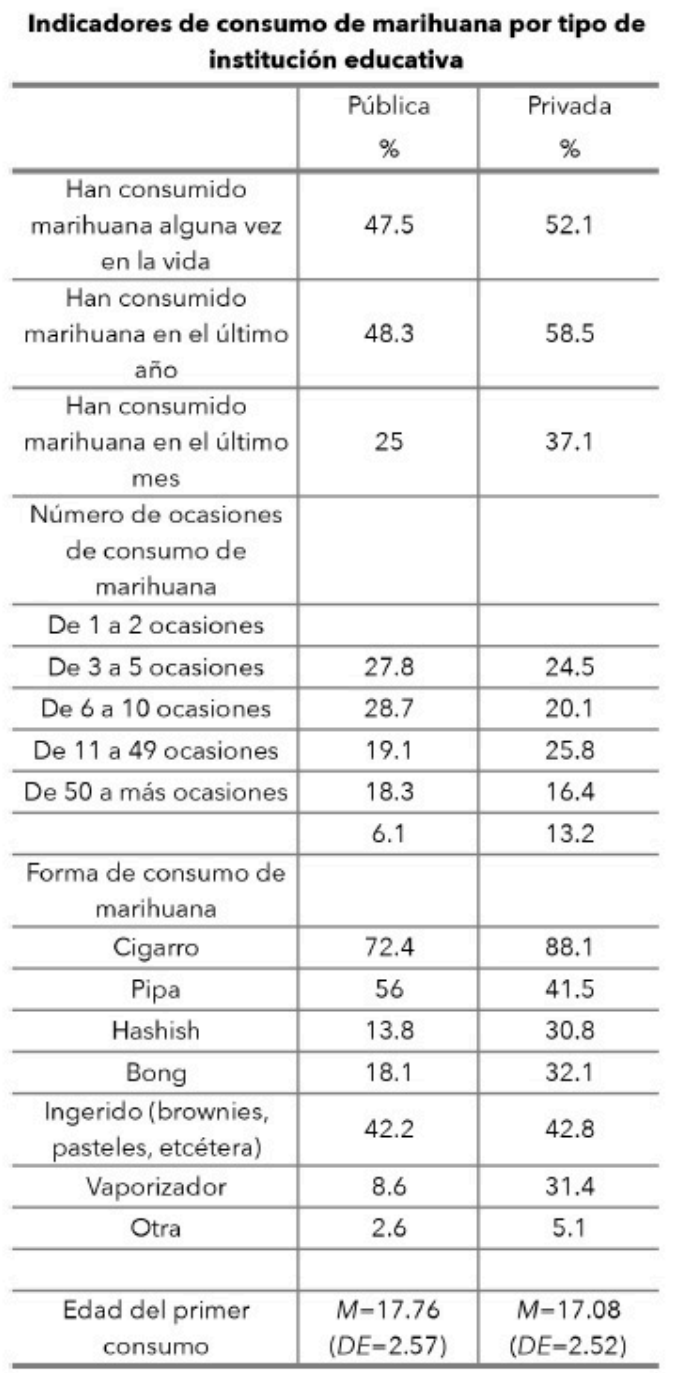

Tabla 2

Indicadores de consumo de marihuana por tipo de institución educativa

En el consumo por hashish, bong y vaporizador fue significativamente mayor el porcentaje de estudiantes de escuelas privadas que el de los universitarios de instituciones públicas $(\mathrm{X} 2=10.77, \mathrm{p}<.001, \mathrm{X} 2=6.77$, $\mathrm{p}<.01$ y X2=20.48, $\mathrm{p}<.001$, respectivamente); en cuanto al consumo por pipa, fue mayor el porcentaje de universitarios de instituciones públicas que informaron que lo consumen que el de privadas $(\mathrm{X} 2=5.67, \mathrm{p}<.05)$. En cuanto al promedio de edad del primer consumo, los jóvenes de instituciones privadas tuvieron un promedio de edad menor que el de los estudiantes de universidades públicas [ $\mathrm{t}(274)=-2.20, \mathrm{p}<.05]$.

\section{Prácticas parentales y consumo de maribuana}

Con base en tres de los indicadores de temporalidad de consumo de marihuana (consumo alguna vez, en el último año y en el último mes) propuestos por Villatoro-Velázquez et al. (2017), se creó una nueva variable que clasificó a los jóvenes en cuatro categorías: los que nunca han 
consumido, los que consumieron alguna vez pero ya no han consumido (consumidores experimentales), los que han consumido en el último año pero no en el último mes, y los que consumieron en el último mes. Cabe señalar que estas cuatro categorías son excluyentes, es decir, un participante no puede estar en más de una categoría. Como se observa en la Figura 1, la mitad de los jóvenes no han consumido marihuana, le sigue en frecuencia los jóvenes que alguna vez consumieron pero no lo han realizado en el año previo al estudio; en tercer lugar se encontraron los estudiantes que han consumido marihuana en el último mes, y con la menor proporción se ubicaron los jóvenes que han consumido dicha sustancia en el último año pero no en el último mes.

Con la clasificación anterior, se realizaron análisis de varianza de una vía para determinar las diferencias entre cada una de las prácticas parentales. Como se puede observar en la Tabla 3, se encontraron diferencias estadísticamente significativas en todas las dimensiones de la mamá, las pruebas post hoc Scheffe indicaron que en el caso de la dimensión de comunicación las diferencias fueron entre los jóvenes que nunca han consumido marihuana y los que lo han hecho en el último mes, donde los primeros fueron quienes percibieron tener una mejor comunicación con su madre, en contraste con los que han tenido consumo en los 30 días previos al estudio. En lo que respecta a la imposición y al control psicológico, los hallazgos indicaron que las diferencias fueron entre los estudiantes que han consumido marihuana en el último mes con los jóvenes de las otras categorías; es decir, que los estudiantes que han consumido dicha sustancia en el último mes perciben una mayor imposición y un mayor control psicológico por parte de su mamá en comparación con el resto de los estudiantes. Respecto al control conductual, las pruebas de múltiples comparaciones no arrojaron diferencias significativas.

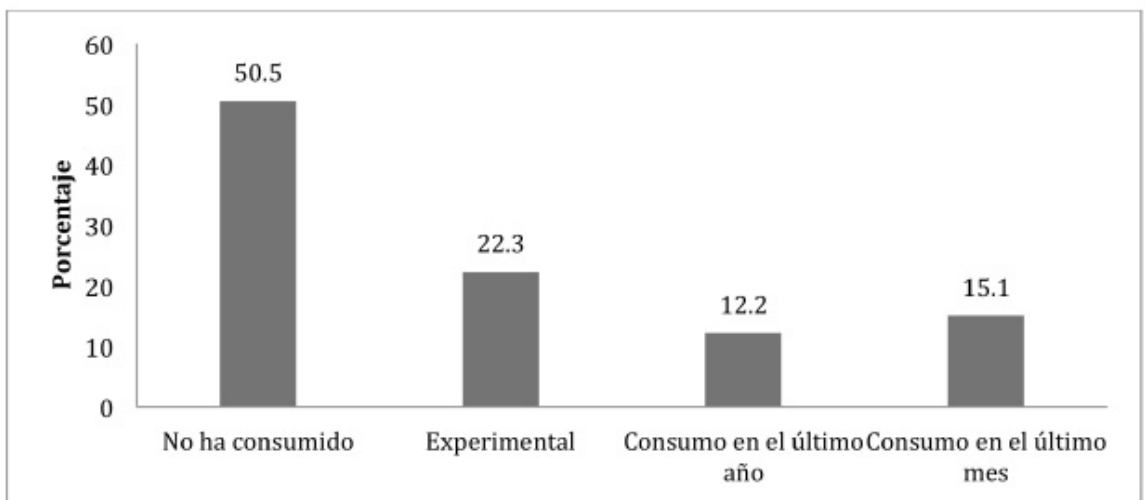

Figura 1

Distribución de los estudiantes por frecuencia de consumo de marihuana

Figura 1

Distribución de los estudiantes por frecuencia de consumo de marihuana

En cuanto a la dimensión de la autonomía, los resultados mostraron diferencias significativas entre los universitarios que han consumido marihuana en el último mes y el resto de los jóvenes, donde, los estudiantes que han consumido en el mes previo al estudio perciben una menor 
promoción de la autonomía por parte de su madre en comparación con el resto de estudiantes (ver Tabla 3).

Tabla 3

Diferencias en las prácticas parentales maternas por categoría de consumo de marihuana

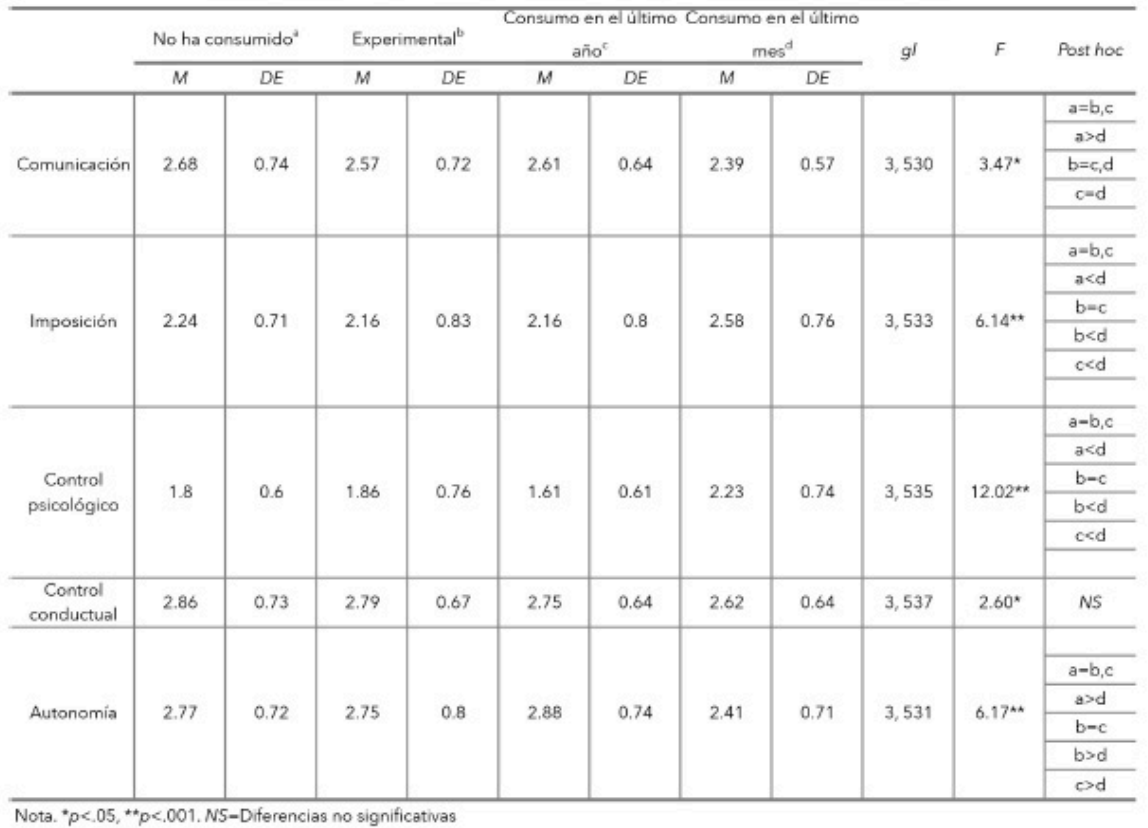

Tabla 3

Diferencias en las prácticas parentales maternas por categoría de consumo de marihuana

Por lo que se refiere a los resultados de las prácticas parentales del padre, éstos mostraron diferencias significativas en casi todas las dimensiones, excepto en el control conductual (ver Tabla 4). En cuanto a la comunicación paterna, los hallazgos de las pruebas post hoc Scheffe indicaron que los jóvenes que nunca han consumido marihuana son quienes perciben que tienen una mejor comunicación con su padre, en contraste con los universitarios que han consumido marihuana en el último mes. Por lo que respecta al control psicológico y a la imposición paterna, los resultados mostraron diferencias significativas entre los jóvenes que han consumido marihuana en el año previo y quienes han consumido en el último mes, donde los primeros son quienes perciben una menor imposición y control psicológico del papá que los han consumido en el último mes. Finalmente, en cuanto a la dimensión de la autonomía paterna los hallazgos mostraron diferencias entre los jóvenes que han consumido marihuana en el último mes respecto a los que nunca han consumido y los que lo han realizado en el último año, es decir, que los estudiantes que han consumido dicha sustancia en el mes previo al estudio, perciben que sus padres promueven menos la autonomía en comparación con los universitarios que nunca han consumido y los que lo han realizado en el último año. 


\begin{tabular}{|c|c|c|c|c|c|c|c|c|c|c|c|}
\hline \multicolumn{12}{|c|}{$\begin{array}{c}\text { Tabla } 4 \\
\text { Diferencias en las prácticas parentales paternas por categoría de consumo de marihuana }\end{array}$} \\
\hline & \multirow{2}{*}{\multicolumn{2}{|c|}{ No ha consumido ${ }^{a}$}} & \multirow{2}{*}{\multicolumn{2}{|c|}{ Experimental $^{b}$}} & \multicolumn{4}{|c|}{ Consumo en el último Consumo en el último } & \multirow{3}{*}{$g l$} & \multirow{3}{*}{$\mathrm{F}$} & \multirow{3}{*}{ Post hoc } \\
\hline & & & & & & & & & & & \\
\hline & M & $D E$ & M & $D E$ & M & $D E$ & M & $D E$ & & & \\
\hline \multirow{4}{*}{ Comunicación } & \multirow{4}{*}{2.41} & \multirow{4}{*}{0.71} & \multirow{4}{*}{2.31} & \multirow{4}{*}{0.68} & \multirow{4}{*}{2.19} & \multirow{4}{*}{0.67} & \multirow{4}{*}{2.17} & \multirow{4}{*}{0.56} & \multirow{4}{*}{3,531} & \multirow{4}{*}{$3.70^{*}$} & $a=b, c$ \\
\hline & & & & & & & & & & & $a>d$ \\
\hline & & & & & & & & & & & $\mathrm{b}=\mathrm{c}, \mathrm{d}$ \\
\hline & & & & & & & & & & & $c=d$ \\
\hline \multirow{3}{*}{ Imposición } & \multirow[b]{3}{*}{2.04} & \multirow{3}{*}{0.74} & \multirow{3}{*}{2.1} & \multirow{3}{*}{0.85} & \multirow{3}{*}{1.86} & \multirow{3}{*}{0.83} & \multirow{3}{*}{2.29} & \multirow{3}{*}{0.9} & \multirow{3}{*}{3,530} & \multirow{3}{*}{$3.73^{*}$} & $a=b, c, d$ \\
\hline & & & & & & & & & & & $\mathrm{b}=\mathrm{c}, \mathrm{d}$ \\
\hline & & & & & & & & & & & $c<d$ \\
\hline \multirow{3}{*}{$\begin{array}{c}\text { Control } \\
\text { psicológico }\end{array}$} & \multirow{3}{*}{1.7} & \multirow{3}{*}{0.77} & \multirow{3}{*}{1.65} & \multirow{3}{*}{0.7} & & & & & & & $a-b, c, d$ \\
\hline & & & & & 148 & 0.61 & 188 & 0.72 & 3.535 & $379^{*}$ & $\mathrm{~b}=\mathrm{c}, \mathrm{d}$ \\
\hline & & & & & 1.40 & 0.01 & 1.00 & & 3,030 & 3.79 & $c<d$ \\
\hline $\begin{array}{c}\text { Control } \\
\text { conductual }\end{array}$ & 2.28 & 0.71 & 2.27 & 0.72 & 2.06 & 0.73 & 2.25 & 0.72 & 3,533 & 1.67 & NS \\
\hline & & & & & & & & & & & $a=b, c$ \\
\hline Autonomía & 2.75 & 0.81 & 2.53 & 0.86 & 2.67 & 0.89 & 2.42 & 0.78 & 3,534 & $8.46^{\star \star}$ & $a>d$ \\
\hline & & & & & & & & & & & $b=c, d$ \\
\hline & & & & & & & & & & & $c>d$ \\
\hline
\end{tabular}

Tabla 4

Diferencias en las prácticas parentales paternas por categoría de consumo de marihuana

\section{Discusión}

En nuestro país, existen pocos datos epidemiológicos del consumo de sustancias ilegales en población universitaria; si bien, la encodat (Villatoro-Velázquez et al., 2017) muestra hallazgos de la población nacional, no hace un análisis específico en población joven. Los datos encontrados en el presente estudio muestran que la proporción de jóvenes universitarios que reportaron que alguna vez en su vida han consumido marihuana es mayor a la reportada por Villatoro et al. (2009); sin embargo, estas diferencias podrían ser explicadas por el tipo de selección de la muestra, ya que en el estudio de Villatoro et al. (2009) se realizó un muestreo probabilístico de una institución pública de la zona metropolitana de la Ciudad de México y el presente estudio seleccionó a los participantes por un muestreo no probabilístico, que si bien la obtención de datos se realizó en diferentes instituciones (privadas y públicas), no garantiza la representatividad de los universitarios de la zona metropolitana.

Del mismo modo que los datos a nivel nacional (Villatoro-Velázquez et al., 2017), en este estudio se corroboró que es mayor la proporción de hombres que reportan el consumo de marihuana que la de mujeres, lo cual se mantiene constante en los tres indicadores evaluados: consumo alguna vez, en el último año y en el último mes. Asimismo, los datos encontrados en la presente investigación mostraron una mayor proporción de universitarios de instituciones privadas que informaron el consumo de marihuana en comparación con los estudiantes de instituciones públicas. Llama la atención este dato, ya que podría estar relacionado con factores culturales vinculados al nivel socioeconómico, es decir, la normalización de conductas no saludables. Pareciera ser que la incorporación de esta práctica forma parte de una dinámica social en estudiantes de estas 
instituciones, las privadas, donde ya no se atraviesa por la crítica social o por la puesta en riesgo de consecuencias graves al saberse de su consumo, lo cual podría aún estar presente en estudiantes de escuelas públicas; no obstante, se recomienda realizar más estudios para poder corroborar dichos hallazgos. Por otro lado, existe evidencia empírica que sugiere la relación entre las prácticas de los padres sobre el consumo de sustancias de los hijos; específicamente sobre el control conductual, Lac y Crano (2009) realizaron un metaanálisis donde encontraron una fuerte relación entre este tipo de control y el bajo consumo de la marihuana en adolescentes. Del mismo modo, el estudio de Lac et al. (2011) mostró que de las prácticas parentales evaluadas por los autores el control conductual fue el predictor más importante para el consumo de marihuana en adolescentes; sin embargo, en la presente investigación, no se encontraron diferencias significativas en el control conductual (tanto materno como paterno) por consumo de marihuana, lo cual difiere de los estudios previos. Esta diferencia en los hallazgos podría deberse al rango de edad del cual se obtuvo información, ya que los estudios previos en su mayoría se enfocan en población adolescente, edad en la cual los padres todavía realizan un mayor monitoreo de las actividades de sus hijos, que va decrementando con la edad. Es importante mencionar, que las prácticas parentales fueron evaluadas en la actualidad y el consumo de marihuana, si bien cubre aspectos actuales, en la mayoría de los casos inicia cuando están cursando estudios de bachillerato, por lo cual podrían no haberse encontrado diferencias significativas. Es así que se recomienda hacer estudios longitudinales que puedan mostrar los cambios en las diferentes etapas de la adolescencia a la juventud, tanto en el consumo de marihuana como en los cambios que se presentan en las relaciones con los padres, así como la relación que existe entre estas variables para determinar si las conductas de los padres son un factor determinante en el consumo de dicha sustancia o existe otro factor (por ejemplo, accesibilidad, presión de pares, etcétera) que pudiera estar explicando la presencia del consumo.

En cuanto al apoyo parental, que involucra aspectos de comunicación y promoción de la autonomía de los jóvenes, los resultados del presente estudio mostraron diferencias significativas, específicamente en la dimensión de comunicación (tanto materna como paterna) los hallazgos indicaron que aquellos jóvenes que nunca han consumido marihuana fueron quienes presentaron mayores puntajes en contraste con los que han consumido en el último mes; resultados que fueron similares a los de la dimensión de autonomía. No obstante, en esta dimensión no sólo se encontraron diferencias entre los grupos extremos (no han consumido en el último mes), sino que también se encontraron diferencias entre los que no han consumido, los consumidores experimentales y los que han consumido en el último, y los consumidores del último mes, donde estos últimos fueron quienes percibieron una menor promoción de la autonomía por parte de sus padres en comparación con los otros grupos. Estos hallazgos concuerdan con lo reportado por investigaciones previas (Cordova et al., 2014; Johnson et al., 2014; Lac et al., 2011; Luk et al., 2015), las cuales aportan información de que aquellos jóvenes que 
perciben una mayor aceptación y una comunicación más frecuente con los padres tienen una menor probabilidad de consumir drogas ilegales, entre ellas la marihuana.

Finalmente, en lo que se refiere al control psicológico, los resultados de la presente investigación mostraron que en el caso de la madre los jóvenes que han consumido marihuana en el último mes son quienes perciben un mayor número de conductas intrusivas de su mamá en comparación con los estudiantes que nunca han consumido, lo hicieron alguna vez o lo hicieron en el año anterior. Por lo que respecta a los resultados del padre, sólo se encontraron diferencias significativas entre los universitarios que lo han consumido en el año previo y en el mes anterior, donde estos últimos son quienes perciben un mayor control psicológico por parte del padre. Estos hallazgos no coinciden con los de estudios previos (por ejemplo, Kincaid et al., 2011), ya que la mayoría de las investigaciones no reportan una relación significativa del control psicológico con el consumo de drogas ilegales, lo cual podría deberse al tipo de muestra utilizada, es decir, la mayoría de los estudios se han realizado en otros contextos donde tal vez ese tipo de estrategias no son tan utilizadas por los padres como en nuestro contexto o bien, pareciera que no son tan importantes para que los jóvenes se involucren en el consumo de sustancias, por lo que para corroborar los presentes hallazgos se recomienda hacer más investigaciones donde se analice el papel del control psicológico parental.

Como se puede apreciar, un número importante de jóvenes ha consumido alguna vez en su vida marihuana. Si bien la proporción de jóvenes que consumen disminuye en el último año, no se debe olvidar que esta sustancia se considera como puerta de entrada para el consumo de otras drogas ilegales, lo cual puede poner en riesgo la salud de los estudiantes. Al respecto Rivera-Olmos y Parra-Bernal (2016) realizaron una revisión sobre los efectos de la marihuana y concluyeron que ésta tiene una gran negatividad desde el punto de vista de la salud pública, además, indican que no existen bases científicas que permitan apoyar su uso medicinal. Dentro de los efectos negativos más importantes que mencionan los autores están efectos destructores en el sistema nervioso central en desarrollo, problemas de aprendizaje en adolescentes, daños en la estructura y funcionamiento cerebrales (Rivera-Olmos \& Parra-Bernal, 2016).

Asimismo, los hallazgos de este estudio mostraron que si bien existen diferentes factores tanto de tipo individual como contextual que se vinculan al consumo de marihuana, las conductas de los padres aún después de la adolescencia juegan un rol importante para que los hijos se involucren en el consumo de sustancias ilegales, por lo que los resultados del presente estudio pueden emplearse como fundamento empírico para el desarrollo de programas de prevención o tratamiento. 


\section{REFERENCIAS}

Andrade, P., \& Betancourt, O. (2008). Prácticas parentales: Una medición integral. En A. Rivera., R. Díaz-Loving, A. Sánchez \& L. Reyes (Eds.), La psicología social en México XII (pp. 561-565). México: Amepso.

Barber, B. K. (1996). Parental psychological control: Revisiting a neglected construct. Child Development, 67(6), 3296-3319. https://doi.org/10.111 $1 / j .1467-8624.1996 . t b 01915 . x$

Becoña, E., Martínez, Ú., Calafat, A., Fernández-Hermida, J. R., Juan, M., Sumnall Mendes, F., \& Gabrhelík, R. (2013). Parental permissiveness, control, and affect and drug use among adolescents. Psicothema, 25(3), 292-298. https://doi.org/10.7334/psicothema2012.294

Bravo de Medina, R., Echeburúa, E., \& Aizpiri, J. (2010). Características psicopatológicas y dimensiones de personalidad en pacientes adultos jóvenes dependientes del cannabis en tratamiento: Un estudio comparativo. Adicciones, 22(3), 245-251.

Brown, B. B., Mounts, N., Lamborn, S. D., \& Steinberg, L. (1993). Parenting practices and peer group affiliation in adolescence. Child Development, 64(2), 467-482. https://doi.org/10.1111/j.1467-8624.1993.tb02922.x

Castellón, A. (2006). Las adicciones son curables. Un enfoque terapéutico actual. México: Diana.

Cogollo-Milanés, Z., Arrieta-Vergara, K., Blanco-Bayuelo, S., Ramos-Martínez, L., Zapata, K., \& Rodríguez-Berrio, Y. (2011). Factores psicosociales asociados al consumo de sustancias en estudiantes de una universidad pública. Revista de Salud Pública, 13(3), 470-479.

Cordova, D., Heinze, J., Mistry, R., Hsieh, H. F., Stoddard, S., Salas-Wright, C. P., \& Zimmerman, M. A. (2014). Family functioning and parent support trajectories and substance use and misuse among minority urban adolescents: A latent class growth analysis. Substance Use \& Misuse, 49(14),1908-1919. https://doi.org/10.3109/10826084.2014.935792

Costa, M. (2015). Rasgos de personalidad que se asocian al consumo regular de cannabis. Informaciones Psiquiátricas, 221(1), 41-55.

Hernández, V. (2007). Motivaciones del estudiante universitario al consumodedrogas (Tesis doctoral). Universidad Autónoma del Estado de Querétaro, México.

Iglesias, V., Cavada, G., Silva, C., \& Cáceres, D. (2007). Consumo precoz de tabaco y alcohol como factores modificadores del riesgo de uso de marihuana. Revista de Saúde Pública, 41(4), 517-522.

Johnson, B., McBride, D., Hopkins, G., \& Pepper, S. (2014). An examination of parent-child relationships and teen substance use: A brief report.Journal of Child \& Adolescent Substance Abuse, 23(4), 210-216. https://doi.org/1 0.1080/1067828X.2013.786926

Kerr, M., \& Stattin, H. (2000). What parents know, how they know it, and several forms of adolescent adjustment: Further support for a reinterpretation of monitoring? Developmental Psychology, 36(3), 366380. https://doi.org/10.1037//0012-1649.36.3.366

Kincaid, C., Jones, D. J., Cuellar, J., \& Gonzalez, M. (2011). Psychological control associated with youth adjustment and risky behavior in African American single mother families. Journal of Child and Family Studies, 20(1), 102-110. https://doi.org/10.1007/s10826-010-9383-6 
King, K. A., Vidourek, R. A., \& Merianos, A. L. (2015). The association between parenting behaviours and marijuana use based on adolescent age. Drugs: Education, Prevention and Policy, 22(4), 334-343. https://doi.org/10.310 9/09687637.2015.1025703

Lac, A., \& Crano, W. D. (2009). Monitoring matters: Meta-analytic review reveals the reliable linkage of parental monitoring with adolescent marijuana use. Perspectives on Psychological Science, 4(6), 578-586. https:/ /doi.org/10.1111/j.1745-6924.2009.01166.x

Lac, A., Unger, J. B., Basáñez, T., Ritt-Olson, A., Soto, D. W., \& BaezcondeGarbanati, L. (2011). Marijuana use among latino adolescents: Gender differences in protective familial factors. Substance Use \& Misuse, 46(5), 644-655. https://doi.org/10.3109/10826084.2010.528121

Luk, J. W., Patock-Peckham, J. A., \& King, K. M. (2015). Are dimensions of parenting differentially linked to substance use across Caucasian and Asian American college students? Substance Use and Misuse, 50(10), 1360-1369. https://doi.org/10.3109/10826084.2015.1013134

Maccoby, E. E., \& Martin, J. A. (1983). Socialization in the context of the family: Parent-child development. En E. M. Hetherington (Ed.), Handbook of child psychology: Socialization, personality and social development (Vol. 4) (pp.1-101). Nueva York, NY, US: Wiley.

Moreno, K. (2003). Drogas: Las 100 preguntas más frecuentes. México: Trillas.

National Institute on Drug Abuse [nida] (2015). La marihuana. Recuperado de https://www.drugabuse.gov/ es/publicaciones/serie-de-reportes/lamarihuana

Palacios, F., Fuertes, J., \& Cabrear, J. (2001). Las drogas: Tópicos, luces y sombras. Madrid, España: Debate.

Patterson, G. R., Reid, J. B., \& Dishion, T. J. (1992). Antisocial boys: A social interactional approach. Eugene, OR, US: Castalia.

Polaino, A., \& De las Heras, J. (2001). Cómo prevenir el consumo de drogas. Madrid, España: Palabra.

Rivera-Olmos, V. M., \& Parra-Bernal, M. (2016). Cannabis: Efectos en el sistema nervioso central. Consecuencias terapéuticas, sociales y legales. Revista Médica del Instituto Mexicano del Seguro Social, 54(5), 626-634.

Smetana, J. G. (2000). Middle-class African American adolescents' and parents' conceptions of parental authority and parenting practices: A longitudinal investigation. Child Development, 71(6), 1672-1686. https://doi.org/10. 1111/1467-8624.00257

Szerman, B. N., \& Peris, D. L. (2008). Cannabis y trastornos de personalidad. En F. Arias \& J. A. Ramos (Eds.), Aspectos psiquiátricos del consumo de cannabis: Casos clínicos (pp. 89-103). Madrid, España: Observatorio Drododependencias.

unodc. (2016). Informe mundial sobre las drogas 2016. Recuperado de https:// www.unodc.org/doc/wdr2016/WDR_2016_ExSum_spanish.pdf

Villatoro, J. A., Moreno, L. M., Gutiérrez, M. L., Bretón, C. M., Medina-Mora, M. E., \& Amador, N. (2009). Consumo de drogas, alcohol, tabaco y sus factores asociados en estudiantes de bachillerato y universitarios. En L. M. Reidl (Ed.), Desarrollo de nuevos modelospara la prevención y el tratamiento de conductas adictivas (pp. 121-140). México: Miguel Ángel Porrúa. 
Villatoro-Velázquez, J., Resendiz-Escobar, E., Mujica- Salazar, A., BretónCirett, M., Cañas-Martínez, V., Soto-Hernández, I., \& MendozaAlvarado, L. (2017). Encuesta nacional del consumo de drogas, alcohol y tabaco 2016-2017: Reporte de drogas. México: Instituto Nacional de Psiquiatría Ramón de la Fuente Muñiz, Instituto Nacional de Salud Pública, Comisión Nacional Contra las Adicciones, Secretaría de Salud. 